\title{
Asymptomatic Bacteriuria and Urinary Tract Infection in Pregnant Women with and without Diabetes Mellitus and Gestational Diabetes Mellitus-A Case-Control Study
}

\author{
Caroline Schneeberger1,2*, Suzanne E. Geerlings33, Jan Jaap H.M. Erwich4, \\ Edwin R. Van Den Heuvel5, Philippa Middleton², Caroline A. Crowther6 \\ ${ }^{1}$ Department of Medical Microbiology, Academic Medical Center (AMC), University of Amsterdam, Amsterdam, The Netherlands \\ ${ }^{2}$ Australian Research Centre for Health of Women and Babies, Robinson Institute (ARCH), University of Adelaide, Adelaide, Australia \\ ${ }^{3}$ Department of Internal Medicine, division of Infectious Diseases, Academic Medical Center (AMC), University of Amsterdam, \\ Amsterdam, The Netherlands \\ ${ }^{4}$ Department of Obstetrics and Gynaecology, University Medical Center Groningen (UMCG), University of Groningen, Gronin- \\ gen, The Netherlands \\ ${ }^{5}$ Department of Mathematics and Computer Science, Technical University Eindhoven, Eindhoven, The Netherlands \\ ${ }^{6}$ The Liggins Institute, University of Auckland, Auckland, New Zealand \\ Email: *c.schneeberger@amc.uva.nl, s.e.geerlings@amc.uva.nl
}

How to cite this paper: Schneeberger, C., Geerlings, S.E., Erwich, J.J.H.M., Van Den Heuvel, E.R., Middleton, P. and Crowther, C.A. (2017) Asymptomatic Bacteriuria and Urinary Tract Infection in Pregnant Women with and without Diabetes Mellitus and Gestational Diabetes Mellitus-A CaseControl Study. Journal of Diabetes Mellitus, 7, 184-194. https://doi.org/10.4236/jdm.2017.73015

Received: June 21, 2017

Accepted: August 14, 2017

Published: August 17, 2017

Copyright $\odot 2017$ by authors and Scientific Research Publishing Inc. This work is licensed under the Creative Commons Attribution International License (CC BY 4.0).

http://creativecommons.org/licenses/by/4.0/ (c) (i) Open Access

\begin{abstract}
Background: Asymptomatic bacteriuria (ASB) and urinary tract infections (UTI) during pregnancy may contribute to adverse pregnancy outcomes. Diabetes mellitus (DM) and gestational diabetes mellitus (GDM) are considered to be important additional risk factor for ASB and UTI during pregnancy. Aims: To investigate differences in prevalence of ASB and incidence of UTI in pregnant women with and without DM and GDM to inform ASB screening and treatment policies. Methods: Data from 214 pregnant women who gave birth during 2010 at the Women's and Children's Hospital, Adelaide, Australia where cases were women with a clinical diagnosis of (G)DM and controls were matched on date of birth. ASB was defined as the growth of at least $10 \mathrm{e} 5$ colony forming units $/ \mathrm{ml}$ of one organism or any presence of group B streptococcus (GBS) at the first urine culture collected during pregnancy without complaints of a UTI. A clinical UTI was diagnosed by the treating physician, in combination with a positive urine culture it was defined as culture-confirmed UTI. Results: No significant differences in prevalence of ASB (5.6\% and 3.7\%; relative risk (RR) 1.50 ; $95 \%$ confidence intervals (CI) 0.44 5.17), incidence of clinical UTI (4.7\% and 11.2\%; RR 0.42; 95\% CI $0.15-1.14$ ) or culture-confirmed UTI (2.8\% and 3.7\%; RR 0.75; 95\% CI 0.17 - 3.27) between pregnant women with and without $(\mathrm{G}) \mathrm{DM}$ were present. No association was found between ASB and UTI. GBS was the most common causative
\end{abstract}


organism of ASB in women with and without DM (66.7\% and 50.0\%). Conclusion: In contrast with earlier research, no significant differences in prevalence of ASB or incidence of UTI was found between pregnant women with and without $(\mathrm{G}) \mathrm{DM}$.

\section{Keywords}

Urinary Tract Infections, Bacteriuria, Diabetes Mellitus, Pregnancy, Antenatal Screening

\section{Introduction}

In the 1960s, Kass and colleagues described the association of untreated asymptomatic bacteriuria (ASB), bacteriuria without symptoms of a urinary tract infection (UTI), with preterm birth and perinatal mortality [1] [2]. However, subsequent studies showed contradictory results. The prevalence of ASB in pregnancy is $2 \%-10 \%$, although prevalence rates up to $40 \%$ have been reported [3] [4]. Earlier studies demonstrated that $30 \%$ to $40 \%$ of pregnant women with untreated ASB developed pyelonephritis [1] [4] [5]. The hypothesis is that anatomic and physiological changes occurring during pregnancy facilitate bacterial growth and ascent of the bacteria to the kidneys [6]. This led to further research and the introduction of ASB screening and treating policies in many countries.

A systematic Cochrane review on the effect of antibiotic treatment for asymptomatic bacteriuria in pregnancy concluded that treatment of ASB reduces pyelonephritis, low birth weight and preterm birth [4]. However the reduction in low birth weight and preterm birth found in this review has to be interpreted with care, given the poor quality of studies [4].

A more recent study in over 5000 pregnant women in the Netherlands, where currently no standard screen-and-treat policy for ASB during pregnancy is in place, found that ASB was not related to preterm birth in women with an uncomplicated singleton pregnancy. Furthermore, they found that the absolute risk of pyelonephritis in pregnant women with untreated ASB is low (2.4\%) [7].

Recent studies have revealed possible adverse effects of maternal antibiotic use during pregnancy including malformations, cerebral palsy and the presence of antibiotic resistant microorganisms in the newborn [8] [9].

In most countries, including Australia, screening and treatment of ASB during pregnancy to prevent adverse pregnancy outcomes is recommended [10]. This is even though the association between ASB and perinatal morbidity have not been clearly established and the whole range of possible side effects of maternal antibiotic use during pregnancy is not wholly identified yet and currently further investigated [4] [9] [10] [11] [12] [13].

Diabetes mellitus (DM) and gestational diabetes (GDM) are considered important additional risk factor for both ASB and UTI in pregnant women [14] [15]. Furthermore the incidence of GDM is increasing in high-income countries such as Australia [16]. Previous studies with mainly small sample sizes have re- 
ported similar or increased prevalence of ASB up to $24 \%$ in pregnant women with DM compared to women without DM [17] [18] [19].

The incidence of symptomatic UTI is higher in pregnant women with DM (3.3\% - 8.8\%) compared to women without DM (1.3\% - 2.3\%) [12] [17] [19] [20]. Pregnant women with ASB more often develop a symptomatic UTI when they have DM compared to those without DM [17].

Many guidelines have been adopted in favor of a screen and treat strategy to prevent complications in pregnant women with ASB [10] [21]. It remains important to challenge this approach because the truly achieved reduction of complications in pregnancy following the screen and treat approach has to be balanced against the side effects of antibiotics for both mother and baby and the unnecessary costs [8] [9] [22] [23].

This study aimed to describe current practice in a developed country, Australia, and to provide more background for ASB screening and treatment policies, especially for high-risk pregnant women with GDM or DM. The specific aims were to investigate differences in prevalence of ASB and incidence of UTI, including causative organisms, in pregnant women with and without DM or GDM.

\section{Methods}

\subsection{Design and Patients}

For this case-control study we used data from pregnant women with DM or GDM (cases) and without DM or GDM (controls) who received antenatal care in the Women's and Children's Hospital in Adelaide, Australia, during 2010. In Australian guidelines routine urine examination during antenatal assessment is advised and was part of standard care in the Women's and Children's Hospital in Adelaide [24] [25].

Cases were randomly selected using systematic sampling with an interval length of two (alternating) on the basis of a list with names of all women who gave birth during 2010 and who were diagnosed with DM or GDM (according to the hospital administration). Controls were randomly collected from a list of women in the same period. To make the two samples comparable, controls with the same birth dates as the cases were selected. Obstetric, demographic and laboratory data were extracted from medical records up to six weeks postpartum. Women were ineligible if they had no available urine culture result, no access to the medical record, a multiple pregnancy, anatomical abnormalities of the urinary tract or pre-existing medical conditions with a known association with UTI except for pregnancy and diabetes. The Hospital Research and Ethics Committee provided ethical approval.

\subsection{Definitions}

ASB was defined as the growth of at least $10 \mathrm{e} 5$ colony forming units $(\mathrm{cfu}) / \mathrm{ml}$ of one organism or any presence of group B streptococcus (GBS) at the first urine culture (screening urine sample) collected during pregnancy without complaints 
of a UTI. A clinical UTI was defined as a clinical diagnosis made by the treating physician (UTI registered by the treating physician in the clinical record); a culture confirmed UTI was defined as a clinical UTI in combination with a positive urine culture. When women were admitted because of a UTI it was considered to be for pyelonephritis. Recurrent UTI (RUTI) was defined following treating physician. Urine cultures with growth less then $10 \mathrm{e} 5 \mathrm{cfu} / \mathrm{ml}$ or with growth of more than two organisms were defined as mixed growth. Urine cultures without growth were defined as negative. Both DM (type 1 and type 2) and GDM were clinical diagnoses. Screening for GDM with a 50 gram oral glucose challenge test between 24 to 28 weeks gestation was part of standard care in the Women's and Children's Hospital in Adelaide during the study period. Small for gestational age (SGA) was defined as birth weight in relation to gestational age smaller than the 10th percentile, appropriate for gestational age (AGA) between the 10th and 90th percentiles and large for gestational age (LGA) greater than 90th percentile [26].

\subsection{Outcomes}

The primary outcome was the prevalence of ASB diagnosed from the first available antenatal urine culture, as most guidelines recommend collecting urine during the first antenatal visit. Other outcomes were the incidence of both clinical and culture confirmed UTI including pyelonephritis, postpartum UTI (up to 6 weeks after birth), and causative uropathogens. Furthermore, the association between ASB and development of UTI was investigated.

The following maternal and neonatal characteristics were assessed: maternal age at birth, parity, race, type of DM (type 1, 2 or DMG), treatment of DM (insulin, oral hypoglycaemics and/or diet), history of recurrent urinary tract infections (RUTI), use of prophylaxis or antibiotics two weeks before first urine culture, mode of birth (normal vaginal birth, operative vaginal birth, caesarean section), gestational age at birth (categorized as $<32,32-37,37-40$ and $>40$ weeks' gestation), gender child, SGA, LGA, AGA, admission to neonatal intensive care unit independent of duration, five minute Apgar score less than seven, neonatal antibiotic use and positive neonatal blood cultures.

\subsection{Statistics}

Data were analyzed using SPSS software for windows, version 19. Fisher's exact test and the Mann-Whitney test were used to calculate differences in characteristics of the women and infants born form women with and without DM. Relative risk (RR) and $95 \%$ confidence intervals (95\% CI) was calculated to estimate differences in binary outcomes. The Mann-Whitney test was used test differences for continuous outcomes.

\section{Results}

\subsection{Maternal and Neonatal Characteristics}

A total of 214 pregnant with DM and GDM $(\mathrm{n}=107)$ and without DM and 
GDM ( $n=107)$ were eligible for analysis (Figure 1$)$. The 107 women with DM and GDM represent around 30\% of the total number of pregnant women with DM who gave birth at the Women's and Children's Hospital during the year. Demographics of both groups were similar (Table 1(a) and Table 1(b)). Of the

Table 1. (a) Maternal characteristics for women with and without DM or GDM; (b) Neonatal characteristics for infants born to women with or without DM.

(a)

\begin{tabular}{|c|c|c|c|c|c|}
\hline & \multicolumn{2}{|c|}{ With DM $(\mathrm{n}=107)$} & \multicolumn{2}{|c|}{ Without DM $(\mathrm{n}=107)$} & \multirow{2}{*}{$\begin{array}{c}\text { P-value } \\
0.021\end{array}$} \\
\hline Age (years) & \multicolumn{2}{|c|}{$31.3 \pm 5.4$} & \multicolumn{2}{|c|}{$29.6 \pm 5.0$} & \\
\hline & & & & & \\
\hline - Caucasian & 55 & $51.4 \%$ & 55 & $51.4 \%$ & \\
\hline - Asian & 43 & $40.2 \%$ & 41 & $38.3 \%$ & 0.963 \\
\hline - Aboriginal & 3 & $2.8 \%$ & 3 & $2.8 \%$ & \\
\hline - Other or mixed & 6 & $5.6 \%$ & 8 & $7.5 \%$ & \\
\hline History of RUTI & 1 & $0.9 \%$ & 4 & $3.7 \%$ & 0.369 \\
\hline Multipara & 29 & $27.1 \%$ & 22 & $20.6 \%$ & 0.336 \\
\hline \multicolumn{6}{|l|}{ Type of DM } \\
\hline - Type 1 & 3 & $2.8 \%$ & - & - & \\
\hline - Type 2 & 6 & $5.6 \%$ & - & - & - \\
\hline - GDM & 98 & $91.6 \%$ & - & - & \\
\hline \multicolumn{6}{|l|}{ Treatment diabetes } \\
\hline Insulin & 18 & $16.8 \%$ & - & - & - \\
\hline Oral hypoglycemics & 40 & $37.4 \%$ & - & - & - \\
\hline Diet & 105 & $98.1 \%$ & - & - & - \\
\hline
\end{tabular}

Note: Figures are numbers and percentages; or mean and standard deviation; ${ }^{a} \mathrm{p}$-value calculated either with Fisher's exact test or Mann-Whitney test. Abbreviations: DM = diabetes mellitus; GDM= gestational diabetes mellitus; RUTI = recurrent urinary tract infection.

(b)

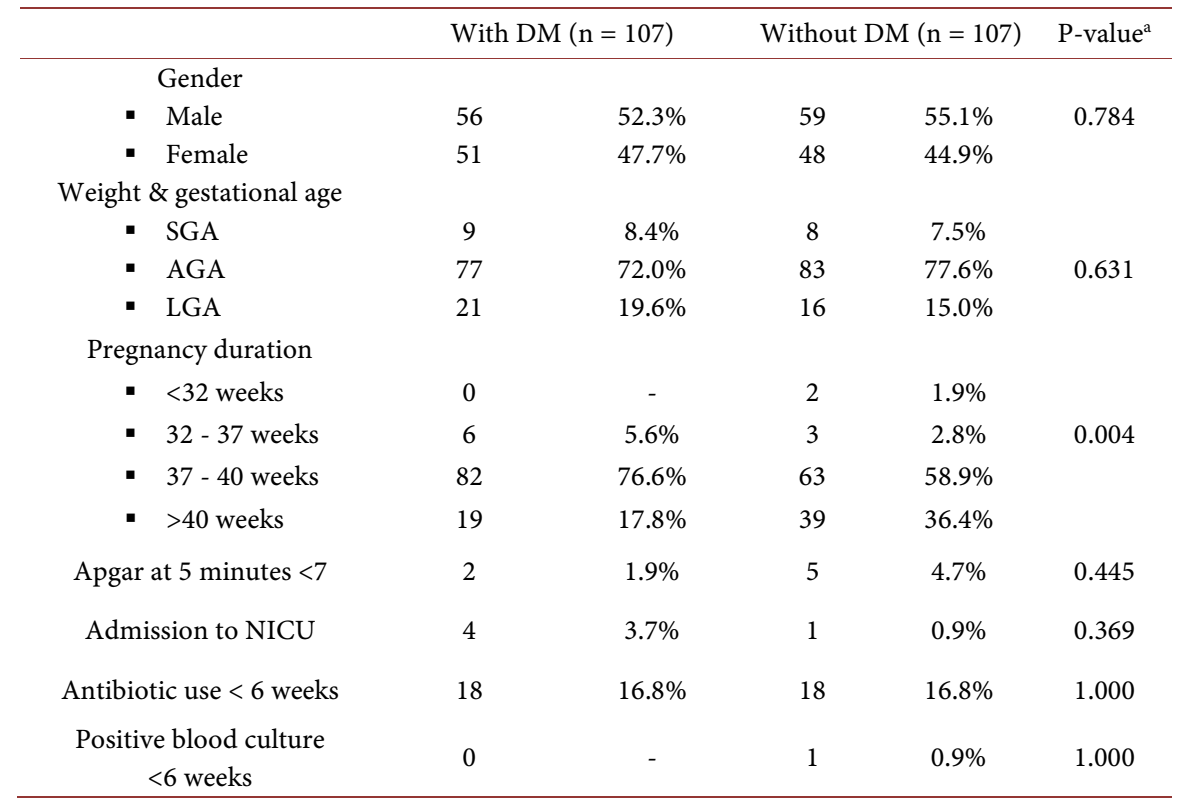

Abbreviations: $\mathrm{DM}=$ diabetes mellitus; $\mathrm{SGA}=$ small for gestational age; $\mathrm{AGA}=$ appropriate for gestational age; LGA= large for gestational age; NICU = neonatal intensive care unit. ${ }^{\mathrm{a}} \mathrm{p}$-values calculated using the Fisher's exact test. 


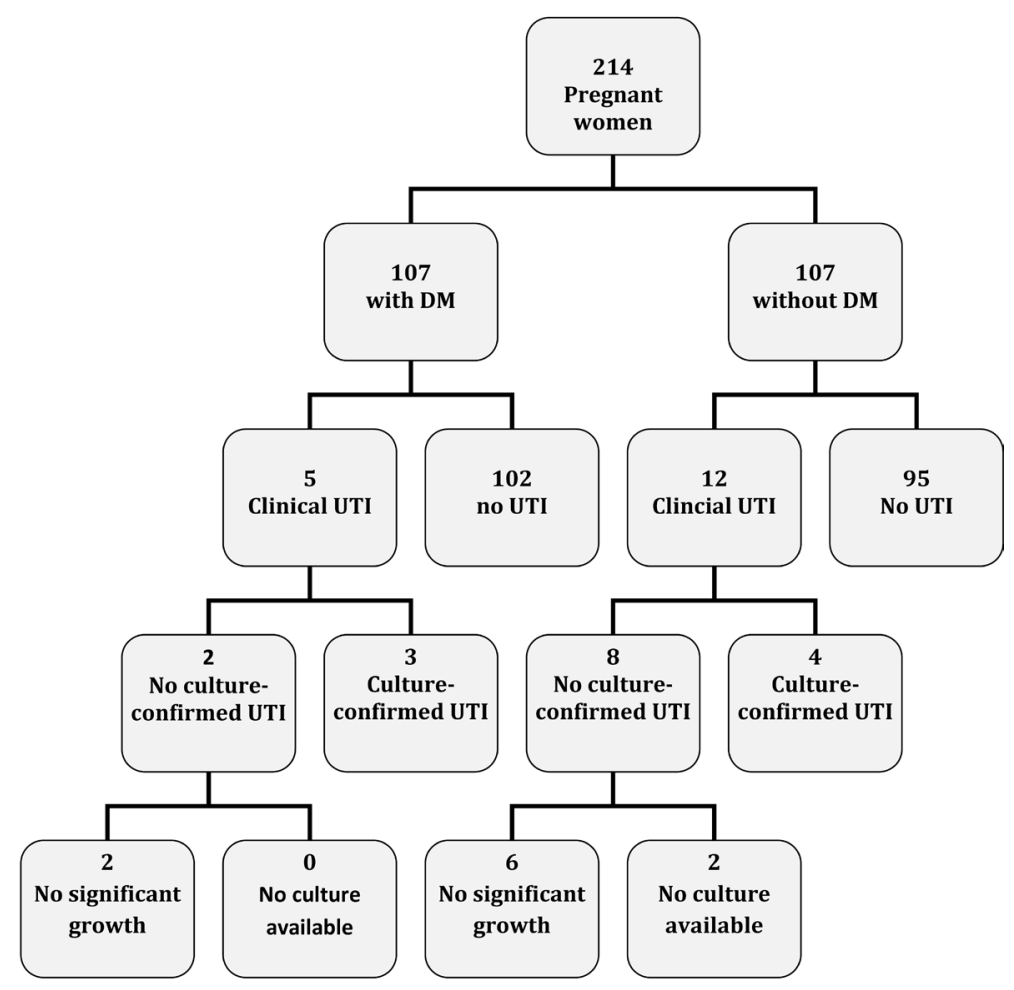

Figure 1. Flow-diagram urinary tract infections in pregnant women with and without $\mathrm{DM}$ and GDM. Abbreviations: $\mathrm{DM}=$ diabetes mellitus; $\mathrm{AB}=$ antibiotics; $\mathrm{GA}$ = gestational age; $\mathrm{ASB}=$ asymptomatic bacteriuria; UTI $=$ urinary tract infection; GBS $=$ Group $\mathrm{B}$ streptococcus. Definitions: ASB was defined as the growth of at least $10 \mathrm{e} 5 \mathrm{cfu} / \mathrm{ml}$ of one or two organisms or any presence of group B streptococcus at the first urine culture collected during pregnancy without complaints of a UTI; Clinical UTI was defined as clinical diagnosis made by the treating physician; Culture confirmed UTI was defined as clinical UTI in combination with a positive urine culture; Positive culture was defined as growth of at least $10 \mathrm{e} 5 \mathrm{cfu} / \mathrm{ml}$ of one or two organisms.

107 women, most were diagnosed with GDM ( $\mathrm{n}=98,91.6 \%)$, of whom 59 $(60.2 \%)$ were treated with diet alone, 33 (33.7\%) received oral hypoglycaemics and $10(10.2 \%)$ used insulin. Part of the women received more than one type of diabetes treatment during their pregnancy. Women without diabetes or GDM more frequently gave birth after 40 weeks of gestation.

\subsection{ASB and UTI}

The mean gestational age at the time of first urine sample was between $11-12$ weeks gestation for both diabetic and non-diabetic women (11.4, Standard deviation $(\mathrm{SD}) \pm 4.2$ and $11.3, \mathrm{SD} \pm 4.5$ weeks). The overall prevalence of ASB was $4.7 \%$ and no differences were found between women with and women without $\mathrm{DM}$ (5.6\% and 3.7\%; relative risk (RR) 1.50; 95\% confidence intervals (CI) 0.44 5.17) (Table 2). Group B Streptococcus (GBS) was the most common causative organism of ASB (60\%) followed by Escherichia coli (E. coli) (30\%). No association was found between ASB and UTI during pregnancy.

One woman with DM (0.9\%) and four (3.7\%) women without DM had symptoms of a UTI at the time of the first urine culture 'the screening urine' and were 
Table 2. Relative risks for urine results in women with and without DM.

\begin{tabular}{|c|c|c|c|c|c|}
\hline & \multicolumn{2}{|c|}{$\begin{array}{l}\text { With DM } \\
(\mathrm{n}=107)\end{array}$} & \multicolumn{2}{|c|}{$\begin{array}{l}\text { Without DM } \\
(\mathrm{n}=107)\end{array}$} & $\begin{array}{l}\text { RR }(95 \% \mathrm{CI}) \\
\text { or p-value }^{\mathrm{a}}\end{array}$ \\
\hline \multicolumn{6}{|c|}{ Antenatal ASB } \\
\hline GA at first urine result (weeks) & \multicolumn{2}{|c|}{$11.4 \pm 4.2$} & \multicolumn{2}{|c|}{$11.3 \pm 4.5$} & 0.609 \\
\hline $\mathrm{AB}$ use $<2$ weeks for "ASB screening" & 2 & $1.9 \%$ & 2 & $1.9 \%$ & - \\
\hline \multicolumn{6}{|l|}{ Results first urine culture "ASB screening" } \\
\hline - $A S B$ without $G B S$ & 2 & $1.9 \%$ & 2 & $1.9 \%$ & \\
\hline - $G B S A S B$ & 4 & $3.7 \%$ & 2 & $1.9 \%$ & 0.5260 \\
\hline - Culture-confirmed UTI & 1 & $0.9 \%$ & 4 & $3.7 \%$ & \\
\hline - No significant growth & 100 & $93.5 \%$ & 99 & $92.5 \%$ & \\
\hline Total ASB including GBS first culture & 6 & $5.6 \%$ & 4 & $3.7 \%$ & $1.50(0.44-5.17)$ \\
\hline \multicolumn{6}{|c|}{ Antenatal UTI } \\
\hline Clinical UTI & 5 & $4.7 \%$ & 12 & $11.2 \%$ & $0.42(0.15-1.14)$ \\
\hline Culture-confirmed UTI & 3 & $2.8 \%$ & 4 & $3.7 \%$ & $0.75(0.17-3.27)$ \\
\hline Received $\mathrm{AB}$ treatment for clinical UTI & 5 & $4.7 \%$ & 11 & $10.3 \%$ & $0.46(0.16-1.26)$ \\
\hline Admission for UTI (pyelonephritis) & 1 & $0.9 \%$ & 1 & $0.9 \%$ & $1.00(0.06-15.78)$ \\
\hline $\begin{array}{l}\text { ASB associated with development of } \\
\text { clinical UTI }(n=10)\end{array}$ & 0 & - & 0 & - & - \\
\hline \multicolumn{6}{|c|}{ Post-partum } \\
\hline Clinical UTI & 3 & $2.8 \%$ & 1 & $0.9 \%$ & $3.00(0.32-28.39)$ \\
\hline Culture-confirmed UTI & 1 & $0.9 \%$ & 1 & $0.9 \%$ & $1.00(0.06-15.78)$ \\
\hline Admission for UTI (pyelonephritis) & 0 & - & 1 & $0.9 \%$ & - \\
\hline
\end{tabular}

Abbreviations: $\mathrm{DM}=$ diabetes mellitus; $\mathrm{AB}=$ antibiotics; $\mathrm{GA}=$ gestational age; $\mathrm{ASB}=$ asymptomatic bacteriuria; UTI = urinary tract infection; GBS = Group B streptococcus; $\mathrm{RR}=$ relative risk; $95 \% \mathrm{CI}=95 \%$ confidence intervals. Definitions: ASB was defined as the growth of at least $10 \mathrm{e} 5 \mathrm{cfu} / \mathrm{ml}$ of one or two organisms or any presence of group B streptococcus at the first urine culture collected during pregnancy without complaints of a UTI; Clinical UTI was defined as clinical diagnosis made by the treating physician; Culture confirmed UTI was defined as clinical UTI in combination with a positive urine culture; Positive culture was defined as growth of at least $10 \mathrm{e} 5 \mathrm{cfu} / \mathrm{ml}$ of one or two organisms. ${ }^{\text {a }} \mathrm{RR}$ and $95 \% \mathrm{CI}$ for binary variables; p-values calculated with Mann-Whitney test for continuous variables.

therefore diagnosed as UTI or pyelonephritis. Only one pregnant woman with ASB received antibiotic treatment. All women with GBS bacteriuria at the first urine culture received antibiotic prophylaxis during birth.

No differences in clinical UTI during pregnancy were seen in pregnant women with and without DM (4.7\% and 11.2\%; RR 0.42; 95\% CI 0.15 - 1.14). In seven of the 17 women with a clinical UTI, a positive culture was present (culture confirmed UTI), in eight women there was a mixed growth, and in two women no culture results were available (Figure 1). Sixteen of the 17 clinical UTIs were treated with antibiotics. Two women (one with and one without DM) were admitted because of a UTI during pregnancy.

No differences in incidence of culture confirmed UTI, including pyelonephritis, during pregnancy $(2.8 \%$ and $3.7 \%$; RR 0.75 ; $95 \% \mathrm{CI} 0.17-3.27)$ and postpartum $(0.9 \%$ and $0.9 \%$; RR 1.00; 95\% CI 0.06 - 15.78) were found between women with and without DM. In both pregnant women with and without DM, E. coli was the most common causative organism of UTI. GBS was not the causative organism for any of the UTIs. 
In both women with and women without diabetes or GDM no association was found between ASB or clinical UTI during pregnancy and adverse pregnancy or neonatal outcomes, including preterm birth and SGA.

\section{Discussion}

In our study, based on clinical practice ASB screening data, we found that the overall prevalence of ASB was rather low (4.7\%). Moreover, no differences in prevalence of ASB or incidence of culture confirmed UTI and clinical UTI was present between pregnant women with and without DM or GDM. Group B streptococcus was the most common causative organism of ASB in both women with and without DM.

No association was seen between ASB or UTI and adverse pregnancy outcomes including preterm birth ( $<37$ weeks) or SGA babies. Although it should be pointed out that the study groups were small and therefore this dataset was only able to detect large differences in pregnancy outcomes.

Little is known about the practical implementation and the efficiency of screening and treating policies for ASB to prevent adverse pregnancy outcomes in pregnant women generally and especially in pregnant women with DM or GDM. The strength of this study is that it gives insight to the practical implementation and the efficiency of the actual screening and treating policy for ASB, including low colony count GBS bacteriuria, in a developed country (Australia) for both women with and without DM.

According to national and international guideline recommendations, there seems to be an under-treatment of ASB in pregnant women in this dataset, in contrast with UTIs. In this study, only for one in ten pregnant women with ASB was antibiotic treatment recorded. A possible explanation for this low treatment rate could be the high percentage of GBS ASB. There are no formal recommendations for management of low colony count GBS bacteriuria during pregnancy; even though GBS bacteriuria may indicate heavy vaginal GBS colonization [18]. In this study we did not find an association between GBS ASB and UTI.

One of the limitations of the study was that only hospital-records and laboratory database were available, while general practitioners could also have diagnosed and treated UTIs contemporaneously, which may not have been recorded in the hospital files. This may partly explain the reduced incidence of UTI in pregnant women with DM and GDM found in this study compared with earlier studies [17]. In addition, all women with GBS bacteriuria at the time of first urine culture received antibiotic prophylaxis during birth. Therefore, it is not possible to make conclusions about the effect of GBS ASB in the postpartum period.

Three earlier studies found either similar or increased percentages of ASB (including GBS ASB) in both pregnant women with DM (4.6\% - 24\%) and pregnant women without DM (4.0\% - 10.9\%) [7] [17] [18] [19]. Only Golan et al. showed an association between ASB and UTI in their prospective cohort study with monthly follow-up including repeating cultures. Among the pregnant women 
with DM and ASB the incidence of UTI was $41.1 \%$ compared to $4.2 \%$ in those without ASB [17].

Nevertheless, in general accurate up-to-date numbers of ASB and UTI in pregnant women including causing organisms are not available due to a variety of reasons. Practice treatment is often started immediately when typical dysuria complaints are present without performing or awaiting for urine culture results. It may be difficult to distinguish between "real" UTI and pregnancy related symptoms, especially in pregnant women. Pregnancy symptoms, such as frequency and abdominal pain, impede the differentiation between ASB and UTI [2]. In order to make a contribution to accurate numbers we separated culture confirmed UTI and clinical UTI. It must be noted that detailed information on symptoms of UTI was often missing in the medical records.

This study reveals that for more than $50 \%$ of the time when antibiotics for UTI were prescribed during pregnancy there was no documented information about a positive urine culture available within the health records. The Australian guidelines state that a urine culture is the golden standard for all suspected UTIs [24] [25]. Performing a culture before commencing treatment should prevent over-treatment by discriminating between "real" UTI and pregnancy symptoms. Recent studies have shown that maternal antibiotic use during pregnancy can be associated with adverse effects for the newborn [8] [9].

\section{Conclusion}

Our study demonstrated that pregnant women with DM or GDM do not have an increased risk for ASB or UTI and that the overall prevalence of ASB in pregnant with and without DM or GDM was low and finally that GBS bacteriuria is common in both pregnant women with and without DM or GDM. Despite the limitations of the retrospective study design, these results are useful input for future discussions regarding ASB screening and treating policies for pregnant women currently in place in developed countries.

\section{Acknowledgements}

We would like to thank the staff of the medical records department of the Women's and Children's Hospital Adelaide for their assistance.

\section{Sources of Financial Support}

1) University Medical Center Groningen (UMCG), Groningen, the Netherlands;

2) Jo Kolk study fund, the Netherlands.

\section{References}

[1] Kass, E.H. (1962) Pyelonephritis and Bacteriuria. A Major Problem in Preventive Medicine. Annals of Internal Medicine, 56, 46-53. https://doi.org/10.7326/0003-4819-56-1-46

[2] MacLean, A.B. (2001) Urinary Tract Infection in Pregnancy. International Journal of Antimicrobial Agents, 17, 273-276. 
[3] Nicolle, L.E. (2003) Asymptomatic Bacteriuria: When to Screen and When to Treat. Infectious Disease Clinics of North America, 17, 367-394.

[4] Smaill, F. and Vazquez, J.C. (2015) Antibiotics for Asymptomatic Bacteriuria in Pregnancy. The Cochrane Database of Systematic Reviews, 7, CD000490. https://doi.org/10.1002/14651858.CD000490.pub3

[5] Kass, E.H. (1960) Bacteriuria and Pyelonephritis of Pregnancy. Archives of Internal Medicine, 105, 194-198. https://doi.org/10.1001/archinte.1960.00270140016003

[6] Grenier, N., Pariente, J.L., Trillaud, H., Soussotte, C. and Douws, C. (2000) Dilatation of the Collecting System during Pregnancy: Physiologic vs. Obstructive Dilatation. European Radiology, 10, 271-279. https://doi.org/10.1007/s003300050043

[7] Kazemier, B.M., Koningstein, F.N., Schneeberger, C., Ott, A., Bossuyt, P.M., de Miranda, E., Vogelvang, T.E., Verhoeven, C.J., Langenveld, J., Woiski, M., Oudijk, M.A., van der Ven, J.E.,Vlegels, M.T., Kuiper, P.N., Feiertag, N., Pajkrt, E., de Groot, C.J., Mol, B.W. and Geerlings, S.E. (2015) Maternal and Neonatal Consequences of Treated and Untreated Asymptomatic Bacteriuria in Pregnancy: A Prospective Cohort Study with an Embedded Randomised Controlled Trial. The Lancet Infectious Diseases, 15, 1324-1333.

[8] Kenyon, S., Pike, K., Jones, D.R., Brocklehurst, P., Marlow, N., Salt, A. and Taylor, D.J. (2008) Childhood Outcomes after Prescription of Antibiotics to Pregnant Women with Spontaneous Preterm Labour: 7-Year Follow-Up of the ORACLE II Trial. The Lancet, 372, 1319-1327.

[9] Bánhidy, F., Acs, N., Puhó, E.H. and Czeizel, A.E. (2007) Pregnancy Complications and Birth Outcomes of Pregnant Women with Urinary Tract Infections and Related Drug Treatments. Scandinavian Journal of Infectious Diseases, 39, 390-397. https://doi.org/10.1080/00365540601087566

[10] Nicolle, L.E., Bradley, S., Colgan, R., Rice, J.C., Schaeffer, A. and Hooton, T.M. (2005) Infectious Diseases Society of America Guidelines for the Diagnosis and Treatment of Asymptomatic Bacteriuria in Adults. Clinical Infectious Diseases, 40, 643-654. https://doi.org/10.1086/427507

[11] Mann, J.R., McDermott, S., Gregg, A. and Gill, T.J. (2009) Maternal Genitourinary Infection and Small for Gestational Age. American Journal of Perinatology, 26, 667672. https://doi.org/10.1055/s-0029-1220792

[12] Mazor-Dray, E., Levy, A., Schlaeffer, F. and Sheiner, E. (2009) Maternal Urinary Tract Infection: Is It Independently Associated with Adverse Pregnancy Outcome? Journal of Maternal-Fetal \& Neonatal Medicine, 22, 124-128. https://doi.org/10.1080/14767050802488246

[13] Chen, Y.K., Chen, S.F., Li, H.C. and Lin, H.C. (2010) No Increased Risk of Adverse Pregnancy Outcomes in Women with Urinary Tract Infections: A Nationwide Population-Based Study. Acta Obstetricia et Gynecologica Scandinavica, 89, 882. https://doi.org/10.3109/00016349.2010.486826

[14] Shah, B.R. and Hux, J.E. (2003) Quantifying the Risk of Infectious Diseases for People with Diabetes. Diabetes Care, 26, 510-513. https://doi.org/10.2337/diacare.26.2.510

[15] Renko, M., Tapanainen, P., Tossavainen, P., Pokka, T. and Uhari, M. (2011) MetaAnalysis of the Significance of Asymptomatic Bacteriuria in Diabetes. Diabetes Care, 34, 230-235. https://doi.org/10.2337/dc10-0421

[16] Schneider, S., Bock, C., Wetzel, M., Maul, H. and Loerbroks, A. (2012) The Prevalence of Gestational Diabetes in Advanced Economies. Journal of Perinatal Medicine, 40, 511-520. https://doi.org/10.1515/jpm-2012-0015 
[17] Golan, A., Wexler, S., Amit, A., Gordon, D. and David, M.P. (1989) Asymptomatic Bacteriuria in Normal and High-Risk Pregnancy. The European Journal of Obstetrics \& Gynecology and Reproductive Biology, 33, 101-108.

[18] Alvarez, J.R., Fechner, A.J., Williams, S.F., Ganesh, V.L. and Apuzzio, J.J. (2009) Asymptomatic Bacteriuria in Pregestational Diabetic Pregnancies and the Role of Group B Streptococcus. American Journal of Perinatology, 27, 231-234. https://doi.org/10.1055/s-0029-1239485

[19] Rizk, D.E., Mustafa, N. and Thomas, L. (2001) The Prevalence of Urinary Tract Infections in Patients with Gestational Diabetes Mellitus. International Urogynecology Journal and Pelvic Floor Dysfunction, 12, 317-321. https://doi.org/10.1007/s001920170033

[20] Harris, R.E. and Gilstrap, L.C. (1981) Cystitis during Pregnancy: A Distinct Clinical Entity. Obstetrics \& Gynecology, 57, 578-580.

[21] U.S. Preventive Services Task Force (2008) Screening for Asymptomatic Bacteriuria in Adults: U.S. Preventive Services Task Force Reaffirmation Recommendation Statement. Annals of Internal Medicine, 149, 43-47. https://doi.org/10.7326/0003-4819-149-1-200807010-00009

[22] Rouse, D.J., Andrews, W.W., Goldenberg, R.L. and Owen, J. (1995) Screening and Treatment of Asymptomatic Bacteriuria of Pregnancy to Prevent Pyelonephritis: A Cost-Effectiveness and Cost-Benefit Analysis. Obstetrics \& Gynecology, 86, 119123.

[23] Wadland, W.C. and Plante, D.A. (1989) Screening for Asymptomatic Bacteriuria in Pregnancy. A Decision and Cost Analysis. The Journal of Family Practice, 29, 372376.

[24] The Royal Australian and New Zealand College of Obstetricians and Gynaecologists (RANZCOG) College Statement C-Obs 3 (a) (2012) Pre-Pregnancy Counselling and Routine Antenatal Assessment in the Absence of Pregnancy Complications.

[25] Australian Health Ministers' Advisory Council (2012) Clinical Practice Guidelines: Antenatal Care-Module 1.

[26] Kloosterman, G.J. (1970) On Intrauterine Growth. International Journal of Gynecology \& Obstetrics, 8, 895-912. https://doi.org/10.1002/j.1879-3479.1970.tb00313.x

\section{Scientific Research Publishing}

Submit or recommend next manuscript to SCIRP and we will provide best service for you:

Accepting pre-submission inquiries through Email, Facebook, LinkedIn, Twitter, etc. A wide selection of journals (inclusive of 9 subjects, more than 200 journals)

Providing 24-hour high-quality service

User-friendly online submission system

Fair and swift peer-review system

Efficient typesetting and proofreading procedure

Display of the result of downloads and visits, as well as the number of cited articles

Maximum dissemination of your research work

Submit your manuscript at: http://papersubmission.scirp.org/

Or contact jdm@scirp.org 